\title{
Preface to the Special Issue on the International Workshop on Applied Mathematics Errachidia (IWAM'2017)
}

\author{
Guest Editors: \\ M. R. Sidi Ammi ${ }^{1}$, M. Tilioua ${ }^{2}$, Delfim F. M. Torres ${ }^{3}$, \\ ${ }^{12}$ FST Errachidia, Moulay Ismaïl Univ., Morocco \\ ${ }^{3}$ Univ. of Aveiro, Portugal
}

\section{Preface to the Special Issue}

The International Workshop on Applied Mathematics, Errachidia (IWAM'2017) took place in Errachidia, Morocco at the Faculty of Sciences and Technics during March 13, 2017 (https://iwam2017.sciencesconf.org/). The workshop was held with the support of FST Errachidia, the University Moulay Ismaïl. It is aimed at celebrating the collaborative research in applied mathematics by the mathematicians of the FST Errachidia, the Systems and Control Group of CIDMA, University of Aveiro, and with the MIA Lab, University of La Rochelle. It was attended by about 100 Mathematicians and Ph.D. and M.S. students, coming from universities from different countries, such as Algeria, Egypt, France, Portugal, and Morocco. The aim of IWAM'2017 is to bring researchers and professionals to discuss recent developments in both theoretical and applied mathematics, to create the knowledge exchange platform between mathematicians. The workshop is broad-based that covers several branches of engineering sciences, mathematics and interdisciplinary researches mainly in the fields of optimization and variational analysis, theoretical, asymptotic and numerical analysis of ordinary, partial and fractional differential equations.

This special issue contains nine original research papers that were carefully selected among works presented during IWAM'2017:

- Error estimates and analysis results for Signorini's problem in thermo-electro-viscoelasticity, by Mohamed Alaoui, El-Hassan Essoufi, Mustapha Bouallala

- Fuzzy a semigroups of operators, by A. El Allaoui, S. Melliani, L. S. Chadli

- Attitude control of a quadcopter platform based on fractional control laws, by T. Ennaciri, N. Mrani, A. El Abbassi, J. Foshi

- A lightweight neural classifier for intrusion detection, by Azidine Guezzaz, Ahmed Asimi, Younes Asimi, Zakariae Tbatou, Yassine Sadqi 
- Binary linear codes and binary matrices, by Driss Harzalla

- Existence of solution of neutral fractional impulsive differential equations with infinite delay, by Khalid Hilal, Youssef Allaoui

- Regional Gradient Exact Enlarged Controllability (G.E.E.C) of the semilinear heat equation, by Touria Karite, Ali Boutoulout, Fatima Zahrae El Alaoui

- Source to coast pollutants distribution in Tangier area: Arc GIS interpolation and finite element methods, by Saiida Lazaar, Siham Abourida

- Duality between differentiability and minimization in Banach spaces, by Nourddin Saidou

\section{Acknowlegdment}

We deeply thank the members of the Scientific and Organizing Committee of IWAM'2017, the participants of the workshop, all the authors for their high quality papers, all those who served in addition as reviewers for this special issue as well as to the editors for their interest in the topics of IWAM'2017 and for having accepted our proposal.

Guest Editors:

M. R. Sidi Ammi, FST Errachidia, Moulay Ismaïl Univ., Morocco M. Tilioua, FST Errachidia, Moulay Ismaïl Univ., Morocco Delfim F. M. Torres, Univ. of Aveiro, Portugal 